\title{
Zur Theorie der Stufenreaktionen, insbesondere bei der Bildung und Verseifung der Dicarbonsäureester
}

\author{
von
}

\section{Rud. Wegscheider,} w. M. k. Akad.

Aus dem I. Chemischen Laboratorium der k. k. Universität in Wien.

(Vorgelegt in der Sitzung am 29. April 1915.)

\section{Historisches und Kritisches.}

Die Theorie der Folgereaktionen ist zuerst von Esson ${ }^{1}$ in zwei Fällen richtig behandelt worden, die sich bei einer Arbeit ergeben hatten, deren Versuche hauptsächlich von Harcourt ausgeführt wurden. Der eine der beiden Fälle ist hier ohne Interesse. Der andere dagegen enthält die Theorie der monomolekularen zweistufigen Reaktion bei Fehlen isomerer Zwischenprodukte als Sonderfall in sich, also jenen Fall, der bei der Veresterung symmetrischer Dicarbonsäuren und der Verseifung ihrer Ester mit einem großen Überschuß des Reagens vorliegt. Esson betrachtet die Reaktionen $A+X \rightarrow B, A+Y \rightarrow C, C+Z \rightarrow D$ (wo $X, Y$ und $Z$ in großem Überschuß) für den Fall, daß die Anfangskonzentration von $C$ Null ist, und gibt die richtige Formel für die Summe der jeweiligen Konzentrationen von $A$ und $C$. Man braucht nur den Geschwindigkeitskoeffizienten der ersten Reaktion gleich Null zu setzen, um den Ausdruck für die zweistufige monomolekulare Reaktion zu erhalten, und zwar gibt Esson's Formel $22^{2}$ die Summe der jeweiligen Konzentrationen von Ausgangs- und Zwischenprodukt.

1 Phil. Trans. London, 156, 216 (1866).

2 p. 220. 
Die Theorie der Verseifung der Ester symmetrischer zweibasischer Säuren durch nicht stark überschüssiges Verseifungsmittel für den Fall des stufenförmigen Verlaufes, also allgemeiner gesagt die Theorie der zweistufigen bimolekularen Reaktion bei Fehlen isomerer Zwischenprodukte hat Ostwald ${ }^{1}$ gegeben. Dann hat Walker ${ }^{2}$ die Theorie der monomolekularen zweistufigen Reaktion ohne isomeres Zwischenprodukt, die ein Sonderfall des Esson'schen Falles ist, abgeleitet; er gibt die Formel für die Konzentration des Endproduktes.

Auf eine unter Umständen eintretende wichtige Vereinfachung machten die Versuche von Geite $1^{3}$ über die Verseifung der Acetine aufmerksam, der ebenfalls die Differentialgleichungen für stufenweisen Verlauf bei Fehlen isomerer $Z$ wischenprodukte richtig ansetzte. $A b e 1^{4}$ hat dann klar gezeigt, daß bei stufenweiser Verseifung der Ester eines dreiwertigen Alkohols die Gleichung einer bimolekularen (beziehungsweise bei stark überschüssigem Verseifungsmittel einer monomolekularen) Reaktion herauskommt, wenn sich die Geschwindigkeitskonstanten der drei Stufen wie $3: 2: 1$ verhalten, und hat auch dieses (selbstverständlich auch für dreibasische Säuren gültige) Ergebnis für den Fall $n$-stufiger Reaktionen verallgemeinert. ${ }^{5}$ Für den Fall des Glykoldiacetats hat etwas später auch J. Meyer ${ }^{6}$ den Zusammenhang zwischen dem Konstantenverhältnis $2: 1$ und dem einfachen Reaktionsverlauf dargetan.

Das wesentliche Erfordernis für das Auftreten dieser Reaktionsvereinfachung hat übrigens schon Petersen ${ }^{7}$ noch vor den Arbeiten Geitel's erkannt. Er stellt die Geschwindigkeitsgleichung für die Esterbildung aus Säure und über-

1 Lehrb. d. allg. Ch., 2. Aufl., II2, 278.

2 Proc. Roy. Soc. Edinburgh, 22, 25 (1900).

3 J. pr. Ch. (2), 55, 429 (1897); 57, 113 (1898).

4. In U1zer-Klimont, Allg. u. physiolog. Chemie der Fette, Berlin, Springer, 1906, p. 220; vgl. auch Z. f. El., 12, 681 (1906).

5 Z. physik. Ch., 58, 558 (1906).

6 Z. f. El., 13, 190 (1907).

7 Z. phys. Ch., 16, 385 (1895). 
schüssigem Alkohol auf und gibt dann als Bedingung für die Gültigkeit dieser Gleichung bei mehrbasischen Säuren an:1 „Für die mehrbasischen Säuren muß weiter vorausgesetzt werden, daß beide - oder alle - Carboxyle sich gleich schnell esterifizieren oder daß eine Carboxylgruppe so große Reaktionsfähigkeit besitzt, daß die Fähigkeit der anderen im Vergleich damit verschwindend klein ist. Wenn dies nicht der Fall ist, werden die Werte für $k$ mit fortschreitender Esterbildung stetig abnehmen.« Der erste von ihm erwähnte Fall ist der der Stufenreaktion mit Geschwindigkeitskonstanten, die der Zahl der reaktionsfähigen Gruppen proportional sind, der zweite entspricht der bekannten van't Hoff'schen Erklärung für den Ablauf von Reaktionen mit niedrigerer Ordnung, als die Bruttogleichung vorhersehen läßt. Nur Petersen's Bemerkung über den Gang der Konstanten ist etwas zu allgemein, wie wir noch sehen werden.

Bei allen diesen Arbeiten wurde (meist stillschweigend) vorausgesetzt, daß die Zwischenstufen nicht in isomeren Formen auftreten, ein Fall, der bei den Glyzerinestern tatsächlich nicht zutrifft. Unter Berücksichtigung des Auftretens von Isomeren habe ich ${ }^{2}$ die Theorie entwickelt; es hat sich ergeben, daß der bi-, beziehungsweise bei großem Überschuß des Reagens monomolekulare Reaktionsablauf bei drei verschiedenen, zum Teil ziemlich verwickelten Beziehungen zwischen den Geschwindigkeitskonstanten eintreten kann, daß aber nur eine davon (und zwar die einfachste) so beschaffen ist, daß auch beliebige Gemische des Ausgangsstoffes und der Zwischenprodukte den einfachen Reaktionsablauf zeigen; auch in diesem einfachsten Fall müssen die Geschwindigkeitskonstanten nicht durchwegs der Zahl der reaktionsfähigen Gruppen proportional sein. Die von mir gegebene Darstellung ist selbstverständlich ohne weiteres auch für die Veresterung dreibasischer Säuren mit zwei gleichen und einer mindestens hinsichtlich der Stellung in der Molekel davon verschiedenen sauren Gruppe und die Verseifung ihrer

1 p. 389 oben.

2 Mon. f. Ch., 29, 93, 109, 233 (1908). 
Ester anzuwenden und schließt auch den Fall der zweibasischen Säuren als Sonderfall in sich ein. Um die Formeln für den letzteren Fall $\mathrm{zu}$ erhalten, braucht man nur in den Formeln für die $\xi, \eta, \zeta k_{1}=k_{2}=k_{11}=a=b_{1}=0 \mathrm{zu}$ setzen und wenn man die Annahme einführen will, daß die $Z$ wischenprodukte anfangs nicht vorhanden sind, außerdem $c_{1}=c_{2}=0 .{ }^{1}$ Diesen Fall (zweistufige Reaktion bei Auftreten isomerer Zwischenprodukte) haben dann v. Pesk off und J. Meyer ${ }^{2}$ behandelt. Wenn diese Autoren es für angemessen finden, ${ }^{3}$ zu sagen, daß bei meiner Erörterung der Glyzeridverseifung nichts prinzipiell Neues herausgekommen sei (was ja in gewissem Sinn von jeder Rechnung gilt, die auf dem Massenwirkungsgesetz und bei Simultanreaktionen auf den von mir ${ }^{4}$ allgemein entwickelten Grundsätzen beruht), so kann ich ihnen diese freundliche Bemerkung mit Bezug auf ihre Arbeit mit mehr Recht zurückgeben. Denn ihre Formeln für die Fälle, wo die Integration ausführbar ist, sind Sonderfälle der von mir gegebenen Formeln und es war schon früher (z. B. aus der Arbeit von Cavalier) bekannt, daß bezüglich der Größenverhältnisse der Konstanten von Stufenreaktionen eine große Mannigfaltigkeit besteht. Überdies sind die Rechnungen von v. Peskoff und Meyer nicht fehlerfrei. Unter anderem ist ihre Gleichung 52 falsch, da sie die Gleichungen 43 und 44 unrichtig addiert haben.

$\mathrm{Da}$ sich auch sonst in der Literatur bisweilen unrichtige Formeln finden, wird es nicht überflüssig sein, für den Fall eines großen Überschusses des Verseifungsmittels, wo die Integration immer möglich ist, die richtigen Formeln zusammenzustellen, wie sie sich durch Spezialisierung meiner Glyzeridgleichungen oder durch direkte Integration ergeben. Die Bezeichnungen ändere ich der Übersichtlichkeit halber.

1 Oder $k_{22}=k_{10}=k_{20}=0$, woraus $y_{22}=x_{1}=x_{2}=0$ folgt und bei anfänglichem Fehlen der Zwischenprodukte außerdem $b_{1}=b_{2}=0$.

2 Z. physik. Ch., 82, 129 (1913).

3 p. 131.

4 Z. physik. Ch., 30, 593 (1899); 35. 5ّ13 (1900). 
Es handelt sich also um Reaktionen entsprechend den Gleichungen ${ }^{1}$

$$
\begin{array}{llll}
M+X \rightarrow M_{1}+Y_{1} & \left(x, k_{1}\right) & M_{1}+X \rightarrow M_{2}+Y_{3} & \left(y, k_{2}\right) \\
M+X \rightarrow M_{1}^{\prime}+Y_{2} & \left(x^{\prime}, k_{1}^{\prime}\right) & M_{1}^{\prime}+X \rightarrow M_{2}+Y_{4} & \left(y^{\prime}, k_{2}^{\prime}\right),
\end{array}
$$

wo $X$ und die $Y$ Molekelkomplexe sind, welche die $M$ nicht enthalten, aber sonst beliebig sind. In Klammern sind die Veränderlichen hinzugefügt, welche die nach den einzelnen Reaktionen umgesetzten Mengen in der Raumeinheit angeben, und die den einzelnen Reaktionen zukommenden Geschwindigkeitskoefnzienten. Für den Fall, daß die Anfangskonzentration von $M A$, die der Zwischenprodukte Null und $X$ in großem Überschuß vorhanden ist, hat man die Differentialgleichungen

$$
\begin{aligned}
d x / d t & =k_{1}\left(A-x-x^{\prime}\right) & d y / d t & =k_{2}(x-y) \\
d x^{\prime} / d t & =k_{1}^{\prime}\left(A-x-x^{\prime}\right) & d y^{\prime} / d t & =k_{2}^{\prime}\left(x^{\prime}-y^{\prime}\right)
\end{aligned}
$$

Bei diesen Bezeichnungen ist

$x+x^{\prime}$ die Konzentrationsabnahme von $M$,

$A-x-x^{\prime}$ die jeweilige Konzentration von $M$,

$x-y$ und $x^{\prime}-y^{\prime}$ die Konzentrationen der beiden $Z w i-$ schenprodukte $M_{1}$ und $M_{1}^{\prime}$,

$y+y^{\prime}$ die Konzentration des Endproduktes $M_{2}$,

$x+x^{\prime}+y+y^{\prime}$ die Konzentrationssumme der $Y$ oder die Konzentrationsabnahme von $X$ (also bei der Veresterung die veresterte Menge in Äquivalenten, bei der Alkaliverseifung der Alkaliverbrauch, allgemein die der Reaktion entsprechende 'Titeränderung für die Raumeinheit).

Aus diesem Ansatz folgt:

\section{Allgemeiner Fall.}

$$
\begin{array}{rlr}
x+x^{\prime} & =A\left[1-e^{-\left(k_{1}+k_{1}^{\prime}\right) t}\right] & 2)^{2} \\
A-x-x^{\prime} & =A e^{-\left(k_{i}+k_{1}^{\prime}\right) t} & 3)^{3}
\end{array}
$$

1 Auf die unrichtige Behandlung dieses Falles durch J. M eyer (Z. physik. Ch., 66, 89 [1909]) wird im folgenden in der Regel nicht Bezug genommen, weil sie als durch die Abhandlung von v. Peskoff und Meyer widerrufen anzusehen ist.

2 Entspricht der Gleichung 50) bei v. Pesk off und Meyer.

3 Entspricht der Gleichung 51) bei v. Peskoff und Meyer. 


$$
\begin{aligned}
& x-y=\frac{k_{1} A}{k_{1}+k_{1}^{\prime}-k_{2}}\left[e^{-k_{2} t}-e^{-\left(k_{1}+k_{1}^{\prime}\right) t}\right] \\
& x^{\prime}-y^{\prime}=\frac{k_{1}^{\prime} A}{k_{1}+k_{1}^{\prime}-k_{2}^{\prime}}\left[e^{\left.-k_{2}^{\prime} t-e^{-\left(k_{1}+k_{1}^{\prime}\right) t}\right]}\right. \\
& y+y^{\prime}=A\left[1+\frac{k_{1} k_{2}+k_{1}^{\prime} k_{2}^{\prime}-k_{2} k_{2}^{\prime}}{\left(k_{1}+k_{1}^{\prime}-k_{2}\right)\left(k_{1}+k_{1}^{\prime}-k_{2}^{\prime}\right)} e^{-\left(k_{1}+k_{1}^{\prime}\right) t}-\right. \\
& -\frac{k_{1}}{k_{1}+k_{1}^{\prime}-k_{2}} e^{-k_{2} t}-\frac{k_{1}^{\prime}}{k_{1}+k_{1}^{\prime}-k_{2}^{\prime}} e^{-k_{2}^{\prime} t} \\
& x+x^{\prime}+y+y^{\prime}= \\
& =A\left[2-\frac{k_{1}}{k_{1}+k_{1}^{\prime}-k_{2}} e^{-k_{2} t}-\frac{k_{1}^{\prime}}{k_{1}+k_{1}^{\prime}-k_{2}^{\prime}} e^{-k_{2}^{\prime} t}-\right. \\
& \left.-\left(2-\frac{k_{1}}{k_{1}+k_{1}^{\prime}-k_{2}}-\frac{k_{1}^{\prime}}{k_{1}+k_{1}^{\prime}-k_{2}^{\prime}}\right) e^{-\left(k_{1}+k_{1}^{\prime}\right) t}\right] \text {. }
\end{aligned}
$$

Wenn die Summe der Konstanten der ersten Stufe gleich ist einer der Konstanten der zweiten Stufe, so werden von den vorstehenden Formeln jene unbrauchbar, welche die Konstanten dieser zweiten Stufe enthalten. Für $k_{1}+k_{1}^{\prime}=k_{2}$ wird z. B.

$$
\begin{aligned}
x-y & =k_{1} A t e^{-k_{2} t} \\
y+y^{\prime} & =A\left[1-\left(\frac{k_{1}-k_{2}^{\prime}}{k_{2}-k_{2}^{\prime}}+k_{1} t\right) e^{-k_{z} t}-\right. \\
& \left.-\frac{k_{1}^{\prime}}{k_{2}-k_{2}^{\prime}} e^{-k_{2}^{\prime} t}\right]
\end{aligned}
$$

$x+x^{\prime}+y+y^{\prime}=$

$$
\begin{aligned}
=A\left[2-\left(\frac{k_{1}+k_{2}-2 k_{2}^{\prime}}{k_{2}-k_{2}^{\prime}}+k_{1} t\right)\right. & e^{-k_{2} t}- \\
& \left.-\frac{k_{1}^{\prime}}{k_{2}-k_{2}^{\prime}} e^{-k_{2}^{\prime} t}\right] .
\end{aligned}
$$

Ist $k_{1}+k_{1}^{\prime}=k_{2}=k_{2}^{\prime}=k$, so hat man

$$
\begin{gathered}
x-y=k_{1} A t e^{-k t} \text { und } x^{\prime}-y^{\prime}=k_{1}^{\prime} A t e^{-k t} \\
y+y^{\prime}=A\left[1-e^{-k t}(1+k t)\right] \\
x+x^{\prime}+y+y^{\prime}=A\left[2-e^{-k t}(2+k t)\right] .
\end{gathered}
$$

1 Die entsprechende Gleichung 52 bei v. Peskoff und Meyer ist falsch. 
Monomolekularer Reaktionsablauf. Er (und ebenso der bimolekulare bei nicht überschüssigem Reagens ${ }^{1}$ ) tritt (abgesehen von Fällen, wo einzelne Konstanten Null werden, und etwaigen Fällen, die an bestimmte Konzentrationen der Anfangsstoffe und Zwischenprodukte gebunden sind) nur für $k_{1}+k_{1}^{\prime}=2 k, k_{2}=k_{2}^{\prime}=k$ auf. Die Gleichheit von $k_{1}$ und $k_{1}^{\prime}$ ist dabei nicht erforderlich. In diesem Fall hat man

$$
\begin{aligned}
& x+x^{\prime}=A\left(1-e^{-2 k t}\right) \quad \text { und } A-x-x^{\prime}=A e^{-2 k t} \\
& x-y=\frac{k_{1} A}{k}\left(e^{-k t}-e^{-2 k t}\right) \text { und } \\
& \qquad x^{\prime}-y^{\prime}=\frac{k_{1}^{\prime} A}{k}\left(e^{-k t}-e^{-2 k t}\right) \\
& y+y^{\prime}=A\left(1+e^{-2 k t-2 e^{-k t}}\right) \\
& x+x^{\prime}+y+y^{\prime}=2 A\left(1-e^{-k t}\right)
\end{aligned}
$$

Keine isomeren Zwischenprodukte. Der Fall geht aus dem allgemeinen durch $k_{1}^{\prime}=k_{2}^{\prime}=0$ hervor. Man hat

$$
\begin{array}{rlrl}
x & =A\left(1-e^{-k_{1} t}\right) & 17)^{2} \\
A-x & =A e^{-k_{1} t} & 18)^{3} \\
x-y & =\frac{k_{1} A}{k_{1}-k_{2}}\left(e^{-k_{2} t}-e^{-k_{1} t}\right) & 19)^{4} \\
y & =A\left(1+\frac{k_{2}}{k_{1}-k_{2}} e^{-k_{1} t}-\frac{k_{1}}{k_{1}-k_{2}} e^{-k_{2} t}\right) & 20)^{5} \\
x+y & \left.=A\left[2-\frac{k_{1}}{k_{1}-k_{2}} e^{-k_{2} t}-\left(2-\frac{k_{1}}{k_{1}-k_{2}}\right) e^{-k_{1} t}\right] \cdot 21\right)^{6}
\end{array}
$$

1 Siehe Wegscheider, Mon. f. Ch., 29, 124 (1908).

2 Die Formel findet sich bei Quartaroli, Gazz. chim., 33, I, 498 (1903).

3 Gleichung 3) bei J. Meyer, Z. physik. Ch., 66, 83 ff. (1909).

4 Gleichung 14) bei Meyer, a. a. O.

${ }^{5}$ Diese Gleichung ergibt sich leicht aus der von Esson angegebenen Formel. Sie findet sich bei Walker (p. 26), Quartaroli (p. 499) und v. Peskoff und Meyer (Gleichung 57). Die entsprechende Gleichung 16 bei Meyer (a. a. O.) ist falsch.

${ }^{6}$ Gleichung 18 bei Meyer. Die von Quartaroli (p. 500) gegebene Formel ist falsch (wahrscheinlich durch unvollständigen Abdruck entstellt). 
Ist $k_{1}=k_{2}=k$, so gelten für $x-y, y$ und $x+y$ die Formeln 11), 12), 13), wobei nur $x^{\prime}=y^{\prime}=0$ und $k_{1}=k$ zu setzen ist.

Der monomolekulare Reaktionsablauf tritt ein für $k_{1}=$ $=2 k_{3}=2 k$. Man hat

$$
\begin{gathered}
x=A\left(1-e^{-2 k t}\right), \quad A-x=A e^{-2 k t}, \\
\left.x-y=2 A\left(e^{-k t}-e^{-2 k t}\right), \quad y=A\left(1+e^{-2 k t}-2 e^{-k t}\right), \quad 22\right)^{1} \\
x+y=2 A\left(1-e^{-k t}\right) .
\end{gathered}
$$

Für diesen Fall ist

$$
y / A=2-x / A-2(1-x / A)^{0.5} .
$$

Der Reaktionsablauf in diesem Fall ist aus folgender Tabelle ersichtlich:



$\begin{array}{llllllllllll}y / A & \ldots & 0.0026 & 0.0112 & 0.0266 & 0.0508 & 0.0858 & 0.1350 & 0.2046 & 0.3056 & 0.4676\end{array}$

$\begin{array}{lllllllllll}(x-y) / A & 0.0974 & 0.1888 & 0.2734 & 0.3492 & 0.4142 & 0.4650 & 0.4954 & 0.4944 & 0.4324\end{array}$

$\begin{array}{llllll}x \mid A & \ldots & 0.95 & 0.99 & 0.995 & 0.999\end{array}$

$y / A \ldots . .0 .6028 \quad 0.8100 \quad 0.8636 \quad 0.9378$

$(x-y) / A \quad 0.3472 \quad 0 \cdot 1800 \quad 0 \cdot 1314 \quad 0.0612$

Man erhält also, wenn man die Reaktion im geeigneten Augenblick unterbricht, ungefähr die Hälfte des Ausgangsstoffes in Form des Zwischenproduktes (z. B. der Estersäure), und zwar, wenn ungefähr drei Viertel des Ausgangsstoffes umgesetzt sind.

\section{Der stufenweise Verlaup bei der Bildung und Verseifung der Dicarbonsäureester.}

Die Kinetik der Veresterung von Dicarbonsäuren und der Verseifung ihrer Ester ist noch wenig bearbeitet worden. Insbesondere gilt das von den aromatischen Dicarbonsäuren. Zum Teil kommt dies daher, daß bei den meisten hierhergehörigen Reaktionen nicht einmal für einbasische Säuren die Theorie

1 Die Formel für $x+y$ findet sich als Gleichung 24 bei Meyer, die für $y$ als Gleichung 58 bei v. Peskoff und Meyer. 
feststeht und wahrscheinlich recht verwickelt ist; es braucht ja nur daran erinnert $z u$ werden, daß auf diesem Gebiet die Theorie der katalytischen und der Neutralsalzwirkung gebraucht wird. Insbesondere hat aber dazu die Schwierigkeit beigetragen, die dadurch entsteht, daß es sich bei Dicarbonsäuren um Reaktionen handelt, die stufenweise verlaufen und daher rechnerisch nicht ganz einfach $z u$ behandeln sind. Daß bei der Veresterung zweibasischer Säuren und der Verseifung ihrer Ester Stufenreaktionen beteiligt sind, bedarf keines kinetischen Beweises; denn das folgt aus der lang bekannten Tatsache, daß man bei der Veresterung dieser Säuren (auch der symmetrischen) oder der Verseifung ihrer neutralen Ester Estersäuren isolieren kann und daß diese selbst wieder veresterbar und verseifbar sind. So hat, um nur an die mir augenblicklich bekannten ältesten Beispiele zu erinnern, Guerin schon $1837^{1}$ gezeigt, daß man beim Erhitzen von Weinsäure oder Traubensäure mit Alkoholen Estersäuren bekommt. Ebenso alt ist die Beobachtung von Malaguti ${ }^{2}$ an der Kampfersäure, daß durch Veresterung mit Alkohol und Schwefelsäure Estersäure entsteht. Durch Veresterung mit Alkohol und Chlorwasserstoff hat Anderson ${ }^{3}$ aus der Hemipinsäure und, um auch eine symmetrische Säure zu nennen, Claus ${ }^{4}$ aus der Dibrombernsteinsäure Estersäuren erhalten. Älter als alle jetzt erwähnten Beobachtungen ist die von Mitscherlich ${ }^{5}$ an der Oxalsäure, daß die Verseifung durch Alkalien in alkoholischer Lösung Estersäuren liefert. Die Bildung von Estersäuren bei der Verseifung mit alkoholischem Chlorwasserstoff habe ich ${ }^{6}$ an der Hemipinsäure beobachtet. Die kinetische Untersuchung derartiger Reaktionen hat also nicht die Aufgabe, zu entscheiden, ob die Reaktion stufenweise verläuft oder nicht, sondern nur die, zu prüfen, ob sich der Reaktionsablauf durch die Annahme der stufenweisen Veresterung, beziehungsweise

1 Ann. Pharm., 22, 248.

2 Ann. Pharm., 22, 32 (1837).

3 Ann. Ch. Ph., 86, 195 (1853).

4 Ber. D. ch. G., 15, 1844 (1882).

5 Pogg. Ann., 33, 332 (1834).

6 Mon. f. Ch., 16, 92 (1895). 
Verseifung restlos darstellen läßt; denn es könnte neben der Stufenreaktion noch eine direkte Bildung des Endproduktes eintreten. Eine darüber hinausgehende Aufgabe könnte der kinetischen Untersuchung nur dann zufallen, wenn man annehmen wollte, daß es trotz der Isolierung der Estersäuren bei zahlreichen Veresterungen und Verseifungen doch einźelne zweibasische Säuren gebe, bei denen diese Reaktionen nicht stufenweise erfolgen. Diese äußerst unwahrscheinliche Annahme könnte allerdings nur durch kinetische Versuche bewiesen werden; durch das Mißlingen von Versuchen, Estersäuren präparativ zu isolieren, könnte sie nicht gestïtzt werden, da kleine Mengen bei der präparativen Aufarbeitung leicht übersehen werden können. Die entsprechende Berücksichtigung positiver präparativer Befunde kann dagegen manche überflüssige Arbeit ersparen.

Für Veresterungsreaktionen zweibasischer Säuren ist der Nachweis, daß sie restlos als. Stufenreaktionen dargestellt werden können, noch in keinem Fall geliefert worden, für Verseifungsreaktionen ihrer Ester nur in drei Fällen. Der erste dieser Nachweise betraf gleich den schwierigeren Fall, daß die Konstanten der beiden Reaktionsstufen nicht in jenem Verhältnis stehen, bei dem ein scheinbar einfacher Reaktionsablauf eintritt; er wurde von Knoblauch ${ }^{1}$ für die Verseifung des Bernsteinsäurediäthylesters durch wässeriges Alkali geführt. Aber auch in dem Falle des Auftretens des scheinbar einfachen Reaktionsablaufes bildet der Nachweis, daß die Reaktion bei großem Überschuß des Reagens streng monomolekular oder mit kleinerer Menge des Reagens streng bimolekular erfolgt, einen Beweis dafür, daß sie restlos als Stufenreaktion zu betrachten ist, vorausgesetzt, daß auch der Beweis dafür geliefert ist, daß weder die erste noch die zweite Stufe der Reaktion praktisch augenblicklich eintritt. Einen solchen Nachweis hat J. Meyer ${ }^{2}$ für die Verseifung der Ester der Bernsteinsäure und Weinsäure durch Salzsäure geliefert.

1 Z. physik. Ch., 26, 96 (1898).

2 Z. physik. Ch., 66, 96 (1909). 
Eine Vermehrung dieser Nachweise, insbesondere auch für den Fall, daß nicht der einfache Reaktionsablauf eintritt, schien erwünscht. Eine Gelegenheit dazu bot sich, als ich aus anderen Gründen Versuche über die Veresterung der Phtalsäure und die Verseifung ihrer Ester ausführen ließ. Es konnte für zwei weitere Reaktionen, die beide nicht den einfachen Reaktionsablauf zeigen, gezeigt werden, daß sie restlos als Stufenreaktionen darstellbar sind, und zwar für die Verseifung des Phtalsäurediäthylesters durch alkoholisches Natron mit geringem Wassergehalt und für eine Veresterungsreaktion, die Veresterung der Phtalsäure durch alkoholischen Chlorwasserstoff. ${ }^{1}$ Diese beiden Reaktionen in alkoholischer Lösung sind auch früher schon an Dicarbonsäuren untersucht worden; aber die Autoren haben sich damit begnügt. bimolekular oder bei Überschuı̂̉ des Reagens monomolekular zu rechnen.

\section{Die Theorie der einfachen Geschwindigkeits- verhältnisse bei Stufenreaktionen.}

Die Tatsache, daß die zum mono-, beziehungsweise bimolekularen Reaktionsablauf führenden Beziehungen zwischen den Geschwindigkeitskonstanten bei Stufenreaktionen wirklich vorkommen, hat zu Erklärungsversuchen aufgefordert. Gerade beim Triacetin, an dem dieses Verhalten zuerst beobachtet wurde, war dies nicht zu erwarten, da seine verseifbaren Gruppen zum Teil nicht gleichwertig sind. Daß schon Petersen die Gleichheit der Reaktionsgeschwindigkeit der einzelnen in der Molekel befindlichen reaktionsfähigen Gruppen als Bedingung für den einfachen Reaktionsablauf erkannt hat, wurde schon erwähnt. Geitel ${ }^{2}$ weist zur Erklärung des einfachen Konstantenverhältnisses bei der Verseifung der Glyzeride durch Salzsäure mit Recht auf die Zahl der reaktionsfähigen Gruppen

1 Näheres in den beiden ungefähr gleichzeit1y mit dieser Mitteilung an dieser Stelle erscheinenden Abhandlungen von Wegs cheider und v. Amann: »Über Esterverseifung durch Alkalien... * und * Kinetik der in Lösungen der Phtalsäure und ihrer Ester in weingeistigem Chlorwasserstoff auftretenden Reaktionen *.

2 J. pr. Ch. (2), 57, 120 (1898). 
und darauf hin, daß nach den ihm bekannten Versuchen ${ }^{1}$ bei dieser Reaktion die Geschwindigkeitskonstanten von der Natur des Alkohols nur wenig abhängen. ${ }^{2} \mathrm{Abel}^{3}$ hat dann den in dem Hinweis Geitel's auf die Zahl der reaktionsfähigen Gruppen steckenden Gedanken deutlicher herausgearbeitet, indem er betonte, daß für das Eintreten der Reaktion bei der alkalischen Verseifung der Acetine nicht das Auftreffen eines Hydroxylions auf die Molekel überhaupt, sondern das Auftreffen auf eine reaktionsfähige Gruppe maßgebend sein werde. Als Bedingung für das Auftreten des einfachen Verhältnisses hat er ferner angegeben, daß jede reaktionsfähige Gruppe den anprallenden Hydroxylionen in gleicher Weise ausgesetzt und gleich reaktionsfähig ist. ${ }^{4}$ Ich $^{5}$ habe gezeigt, daß der einfache Reaktionsablauf bei Stufenreaktionen auch unter Bedingungen eintreten kann, wo das Verhältnis der Geschwindigkeitskonstanten nicht der Zahl der reaktionsfähigen Gruppen proportional ist (ein Fall, der wohl kaum durch einfache molekulartheoretische Vorstellungen erklärt werden kann), und darauf hingewiesen, daß für das Auftreten von der Zahl der reaktionsfähigen Gruppen proportionalen Geschwindigkeitskonstanten auch erforderlich ist, daß durch die bei der Bildung des Zwischenproduktes eintretende Änderung der Molekel die Reaktionsfähigkeit der einzelnen Gruppen nicht geändert wird und daß dies wahrscheinlich wohl nie genau zutreffen wird. Es ist also beispielsweise bei der Verseifung von Dicarbonsäureestern für das Auftreten der einfachen Konstantenverhältnisse erforderlich, daß die Realstionsfähigkeit einer veresterten Carboxylgruppe durch eine zweite Carboxylgruppe in gleicher Weise beeinfluft wird, gleichgültig ob diese zweite Carboxyl-

1 Vgl. dazu Wegscheider, Mon. f. Ch., 29, 104 (1908).

2 Der Bemänglung dieser Geitel'schen Auffassung durch Abel (U1zerKlimont, Fetie, p. 234) konnte ich mich nicht anschließen (Mon. f. Ch., 29, $91[1908])$.

3 Z. f. El., 12, 681 (1906).

4. Die Gleichheit der Reaktionsfähigkeiten an den primären und sekundären Hydroxylen des Triacetins wollte eben Geitel durch den Hinweis auf den geringen Einfluß der Natur des Alkohols auf die Verseifungsgeschwindigkeit durch Salzsäure begründen.

5 Mon. f. Ch., 29, 94, 91 (1908). 
gruppe verestert oder frei ist. Infolge dieser Erwägung habe ich clas Auftreten des einfachen Konstantenverhältnisses als einen Ausnahmefall betrachtet. Dann hat sich J. Meyer ${ }^{1}$ mit der Frage beschäftigt. Er bemängelt die Abel'sche Darlegung, nach meiner Meinung mit Unrecht; denn er hat in der Tat nur die Abel'sche Auffassung ausführlicher dargelegt. Er sieht aber dabei von den von Abel und mir gemachten Einschränkungen $a b^{2}$ und kommt daher zu dem Schluß, daß bei symmetrischen Substanzen das einfache Konstantenverhältnis auftreten müsse. Die seiner Auffassung widersprechenden Arbeiten von Knoblauch und Cavalier fertigt er mit kurzen Worten ab, ${ }^{3}$ obwohl die letztere Arbeit sich sogar ebenfalls auf die Verseifung durch Wasser bezog. Bald mußte sich aber Meyer ${ }^{4}$ von der schon durch Knoblauch bekannten Tatsache überzeugen, daß seine Annahme bei der alkalischen Verseifung der Dicarbonsäureester nicht zutrifft. Diese Abweichung von seiner Erwartung erklärt er unter Benutzung eines bekannten, zuerst von Ostwald ausgesprochenen Gedankens dadurch, daß bei der Verseifung der Acetate mehrwertiger Alkohole elektrisch neutrale Zwischenprodukte entstehen, bei der Verseifung der Dicarbonsäureester dagegen Anionen, welche andere Anionen, also auch die bei der alkalischen Verseifung reagierenden Hydroxylionen abstoßen. Daß die elektrostatische Abstoßung durch die Ionen bei der Verseifung durch Alkalien eine Rolle spielt, ist schon früher von van't Hoff $^{5}$ auf Grund der Beobachtung von E. Fischer ${ }^{6}$ angenommen worden, daß die salzbildenden Ester schwerer verseift werden als ähnliche nicht salzbildende. ${ }^{7}$

Gegen die Annahme Geitel's, daß die Zahl der reaktionsfähigen Gruppen in manchen Fällen für das Verhältnis der

1 Z. physik. Ch., 66, 115 (1909).

2 Hierzu wurde er durch seine Beobachtung verleitet, daß bei der Verseifung der Ester symmetrischer Dicarbonsäuren durch wässerige Salzsäure das einfache Konstantenverhältnis auftritt.

3 p. 82.

4 Z. physik. Ch., 67, 257 (1909),

5 Ber. D. ch. G., 31, 3277 (1898).

6 Ebendort, p. 3274.

7 Vgl. dazu Goldschmidt und Scholz, Ber. D. ch. G., 40, 636 (1907). 
Geschwindigkeitskonstanten der Ausgangs- und Zwischenstoffe maßgebend ist, gegen die kinetische Deutung dieser Auffassung durch Abel und gegen die Auffassung Meyer's, daß das verschiedene Verhalten der Ester symmetrischer Dicarbonsäuren bei der sauren und alkalischen Verseifung in wässeriger Lösung mit der abstoßenden Wirkung von Anionen auf Hydroxylionen im Zusammenhang stehe, ${ }^{1}$ ist wohl derzeit kein Einwand $\mathrm{zu}$ erheben. ${ }^{2}$ Letztero Annahme erklärt recht gut, warum bei der Verseifung durch Wasser die Reaktionsfähigkeit eines veresterten Carboxyls durch eine veresterte und eine freie Carboxylgruppe ungefähr gleich stark beeinflußt wird, bei der alkalischen Verseifung dagegen nicht. Aber daraus folgt nicht, daß in allen Fällen, wo dieser elektrostatische Einfluß fehlt, bei symmetrisch gebauten Stoffen die Reaktionsgeschwindigkeit der Ausgangs- und Zwischenstoffe immer der Zahl der in der Molekel vorhandenen gleichartigen reagierenden Gruppen proportional ist. Daß vielmehr, entsprechend dem von mir hervorgehobenen Gesichtspunkt, die Reaktionsfähigkeit einer umwandlungsfähigen Gruppe eine Veränderung erleiden kann, wenn sich an einer zweiten gleichartigen Gruppe in der Molekel die Umwandlung vollzieht, ist schon durch die Beobachtungen von Cavalier wahrscheinlich gemacht. Ebenso zeigt der Gang der einstufig nach Äquivalenten gerechneten Konstanten bei verschiedenen Reaktionen, daß bei ihnen wahrscheinlich nicht das einfache Konstantenverhältnis auftritt. ${ }^{3}$ Und auch v. Peskoff und J. Meyer ${ }^{4}$ haben sich bei ihren Versuchen über die saure Verseifung der Amide zweibasischer Säuren nicht der Ansicht verschließen können, daß da die Verhältnisse ganz anders liegen als bei der Verseifung der Säureester.

1 Hierzu hat sich auch Smith (Z. physik. Ch., 81, 340 [1913]) zustimmend geäußert.

2 Immerhin ist es keineswegs sicher, daß bei der Verseifung durch wässerige Säuren elektrostatische Wirkungen nicht in Betracht kommen. Bei der sauren Verseifung der Säureamide sehen sich v. Peskoff und Meyer genötigt, eine solche Wirkung anzunehmen.

3 Siehe den folgenden Abschnitt.

4 Z. physik. Ch., 82, 167 (1913). 
Seither hat Kailan ${ }^{1}$ auch die Veresterung mit Alkohol und Chlorwasserstoff an einigen Dicarbonsäuren der Fettreihe nach dieser Richtung untersucht. Wenn die einstufig monomolekular nach Äquivalenten gerechneten Konstanten in absolutem Alkohol nicht stärker sinken, als durch die Wasserbildung bei der Reaktion erklärt werden kann (Fall der Bernsteinsäure, Weinsäure, Glutarsäure, Malonsäure), so nimmt er an, daß jenes Konstantenverhältnis der beiden Stufen $(2: 1)$ auftritt, bei dem der Gesamtverlauf der Reaktion sich monomolekular darstellen läßt. Nur bei Äpfelsäure und Oxalsäure sinken die Konstanten stärker, woraus der Schluß gezogen wird, daß bei ihnen die Veresterung der ersten Stufe rascher erfolgt, als der Zahl der freien Carboxyle entspricht. Demgemä乃 würde das Konstantenverhältnis $2: 1$ bei symmetrischen Säuren nur dann nicht auftreten, wenn die beiden Carboxyle direkt aneinander gebunden sind. Doch gibt er an, daß nach im Gang befindlichen Versuchen auch bei der Maleinsäure das Konstantenverhältnis nicht $2: 1$ ist. Es ist klar, daß diese indirekte Methode keinen scharfen Beweis für das Konstantenverhältnis $2: 1$ liefern kann. Überdies ist der Schluß auf das Vorliegen dieses Verhältnisses darum bedenklich, weil bei Kailan's Versuchen das Absinken der Konstanten meist auch bei Wassergehalten des Alkohols eintrat, wo Benzoesäure kein deutliches Absinken mehr zeigte. Wenn aber das Absinken nicht auf die Änderung des Wassergehaltes oder eine sonstige Störung zurückgeführt werden kann, *so ist es, wie im folgenden gezeigt wird, ein Beweis dafür, daß das Konstantenverhältnis größer als $2: 1$ ist.

Während somit die Versuche Kailan's zum Teil dafür sprechen, daß bei der Veresterung mit alkoholischem Chlorwasserstoff die Geschwindigkeitskonstanten nicht der Zahl der reaktionsfähigen Gruppen proportional sind und in keinem Fall als sicherer Beweis für eine solche Proportionalität betrachtet werden können, haben meine schon erwähnten, mit v. Amann durchgeführten Untersuchungen über die Veresterung der Phtalsäure den unzweifelhaften Beweis dafür

1 Z. physik. Ch., 85, 706 (1913). 
erbracht, daß bei dieser Säure die Proportionalität zwischen Geschwindigkeitskonstanten und Zahl der reaktionsfähigen Gruppen auch nicht annähernd zutrifft. Vielmehr ist die Konstante der ersten Stufe 13- bis 20 mal so groß als die der zweiten. Dies ist eine schlagende Widerlegung der Ansicht von J. Meyer, daß die Proportionalität zwischen Geschwindigkeitskonstanten und Zahl der reaktionsfähigen Gruppen immer auftritt, wenn nicht elektrostatische Verhältnisse Abweichungen bedingen. Bei der Veresterung der Phtalsäure kommen wahrscheinlich elektrostatische Verhältnisse überhaupt nicht in Betracht, da weder die Säure noch die Estersäure in stark chlorwasserstoff haltigen alkoholischen Lösungen merklich dissoziiert sein dürften ${ }^{1}$ und weil nach dem gegenwärtigen Stande der Theorie dieser Reaktion nicht bezweifelt werden kann, daß undissoziierte Chlorwasserstoffmolekeln, beziehungsweise ihre Verbindungen mit Alkohol dabei eine große (bei den von v. Amann und mir behandelten ziemlich konzentrierten Lösungen wahrscheinlich eine überwiegende) Rolle spielen. Würde man aber selbst annehmen, daß die Reaktion sich wesentlich zwischen Säureionen und Wasserstoffionen, beziehungsweise deren Verbindungen mit Alkohol abspielt, so würde der elektrostatische Effekt darin bestehen, daß die organischen Anionen Wasserstoffionen anziehen und dadurch die Reaktion bewirken. In den einwertigen Phtalsäureionen würde dann nur ein Carboxyl reaktionsfähig sein. Die Geschwindigkeitskonstanten der einwertigen Phtalsäure- und der Phtalestersäureionen müßten daher gleich sein; ihr Wert sei $k$. Die Konstante der zweiwertigen Phtalsäureionen wäre dann nach der Meyer'schen Anschauung $2 k$. Von der gesamten Phtalsäure sei der Bruchteil $\alpha$ als einwertige, $\beta$ als zweiwertige Ionen vorhanden; der Dissoziationsgrad der Phtalestersäure sei $\gamma$. Dann wären die Geschwindigkeitsgleichungen (bei Vernachlässigung der Gegenreaktionen) in folgender Form anzusetzen:

$$
\begin{aligned}
& d x / d t=k(A-x) \alpha+2 k(A-x) \beta=k(A-x)(\alpha+2 \beta) \\
& d y / d t=k(x-y) \gamma .
\end{aligned}
$$

1 Die Dissoziationskonstante der Phtalsäure in alkoholischer Lösung ist von Godlewski (Chem. Zentr., 1904, II, 1275) gemessen worden. 
Die scheinbare Konstante der ersten Stufe ist dann $k(\alpha+2 \beta)$, die der zweiten $k \gamma$.

Die Dissoziationskonstanten der zweiten Stufe der Phtalsäuredissoziation und die der Phtalestersäure in alkoholischer Lösung sind nicht bekannt. Man wird aber als Näherungsannahme immerhin die einführen dürfen, daß die Verhältnisse der Dissoziationskonstanten untereinander von denen in wässeriger Lösung nicht stark abweichen. Nun ist nach Smith $^{1}$ die erste Dissoziationskonstante der Phtalsäure in wässeriger Lösung $700 \mathrm{mal}$ so groß als die zweite. Bei sehr geringer Dissoziation kann man dieses Verhältnis gleich $\alpha .2 / \beta$ setzen, ${ }^{2}$ also $\beta=\alpha^{2} / 700$. Da $\alpha$ selbst sehr klein ist, kann $2 \beta$ neben $\alpha$ vernachlässigt werden. Die erste Dissoziationskonstante der Phtalsäure $(2 K)$ in wässeriger Lösung ist ungefähr doppelt so groß als die der Estersäure. ${ }^{3}$ Daher hat man $2 K=\alpha\left[\mathrm{H}^{\cdot}\right] /(1-\alpha), K=\gamma\left[\mathrm{H}^{\cdot}\right] /(1-\gamma)$, und, indem man $\alpha$ und $\gamma$ neben 1 vernachlässigt, $2=\alpha / \gamma$. Das Verhältnis der Geschwindigkeitskonstanten der ersten und zweiten Stufe wird also nach der Meyer'schen Auffassung $(\alpha+2 \beta) / \gamma \sim 2$. Das wirklich gefundene Konstantenverhältnis ist also durch diese Auffassung durchaus nicht erklärbar.

Besser entspricht der Proportionalität zwischen Geschwindigkeitskonstanten und Zahl der reaktionsfähigen Gruppen in der Molekel die Verseifung des Phtalsäurediäthylesters durch alkoholisches Natron; sie zeigt aber doch auch eine deutliche Abweichung davon. ${ }^{4}$ Auch die älteren Versuche über die Verseifung von Dicarbonsäureestern durch alkoholisches Alkali machen Abweichungen von dieser Proportionalität wahrscheinlich. Immerhin sind bei dieser Reaktion in alkoholischer Lösung die Abweichungen von der Proportionalität bei weitem

1 Z. physik. Ch., 25, 260 (1898). Wenn die Berechnung von Smith auch nicht einwandfrei ist (vgl. Wegscheider, Mon. f. Ch., 23, 642 (1902) so ist doch die Größenordnung sicher. (1902).

2 Vgl. Gleichung 1 und 2 bei Wegscheider, Mon. f. Ch, 23, 600

3 Vl. Wegscheider, Mon. f. Ch., 16, 153 (1895); 23, 346 (1902).

4 Die nähere Erörterung der hier vorliegenden verwickelten Verhältnisse siehe in der Abhandlung: » Uber Esterverseifung durch Alkalien. s 
nicht so groß als in wässeriger Lösung. Hierin liegt eine Stütze für die Annahme, daß elektrostatische Wirkungen bei den Abweichungen eine Rolle spielen können; denn die Reaktion in alkoholischer Lösung ist, wie ich an der angegebenen Stelle zeige, wenigstens bei der Phtalsäure in der Hauptsache eine Reaktion zwischen undissoziierten Molekeln, bei der also elektrostatische Wirkungen keine große Rolle spielen können.

Aus diesen Darlegungen ergibt sich, daß die Annahme von J. Meyer, daß die Proportionalität der Konstanten der einzelnen Reaktionsstufen mit der Zahl der reaktionsfähigen Gruppen in der Molekel die Regel sei, die nur in besonderen Fällen' Ausnahmen erleidet, nicht zutrifft. Es bleibt nach wie vor wahrscheinlich, daß die durch das Eintreten der ersten Reaktionsstufe gesetzte Veränderung der Molekel die Reaktionsfähigkeit der zweiten Gruppe in der Regel verändert. Wenn trotzdem bei einer beträchtlichen $Z$ ahl von Reaktionen ungefähr der der Proportionalität zwischen Geschwindigkeitskonstanten und Zahl der reaktionsfähigen Gruppen entsprechende einfache Reaktionsverlauf beobachtet wird, so darf dies keineswegs als Beweis dafür betrachtet werden, daß durch die Veränderung der ersten Gruppe die Reaktionsfähigkeit der zweiten nicht geändert werde. Denn diese Änderungen machen sich nur dann als beträchtliche Abweichungen von den Gesetzen des einfachen Reaktionsablaufes bemerkbar, wenn sie sehr bedeutend sind. Weicht das Verhältnis der Konstanten von dem für den einfachen Reaktionsablauf erforderlichen nur mäßig $a b$, so sind die Abweichungen von den Gesetzen des einfachen Reaktionsablaufes unbedeutend, wie ich schon bei anderer Gelegenheit gezeigt habe. ${ }^{1}$

IV. Der Gang der bimolekular einstufig gerechneten Konstanten bei bimolekularen zweistufigen Reaktionen.

Bei den bisherigen Versuchen über bimolekulare zweistufige Reaktionen haben sich die Verfasser nicht selten darauf beschränkt, die Konstanten nach den Formeln zu

1 Mon. f. Ch., 29, 98 (1908). 
rechnen, die für bimolekulare einstufige Reaktionen zu erwarten sind. Es ist daher nicht ohne Interesse, zu untersuchen, welcher Gang bei diesen Konstanten $z \mathrm{u}$ erwarten ist. Diesbezüglich liegen zwei verschiedene Rechnungsarten vor. Ein Teil der Verfasser hat in die Gleichungen der einstufigen Reaktion die Konzentration des Stoffes, der stufenweise umgewandelt wird, in Äquivalenten eingesetzt. Ein anderer Teil aber hat die Anfangskonzentration dieses Stoffes in Molen eingesetzt und davon den umgewandelten Betrag in Äquivalenten abgezogen. Der zu erwartende Gang der einstufig gerechneten Konstante ist bei den beiden Rechnungsarten ein verschiedener. Im folgenden wird vorausgesetzt, daß das Zwischenprodukt nicht in Isomeren auftritt.

Drückt man die Konzentration des Ausgangsstoffes in Äquivalenten aus und rechnet bimolekular einstufig, so setzt man statt der richtigen Differentialgleichungen die Gleichung $d(x+y) / d t=k(2 A-x-y)(B-x-y)$ voraus, wo $B$ die Anfangskonzentration des Reagens ist und $k$ die einstufig gerechnete Konstante. Diese Rechnungsweise drückt die Vorstellung aus, daß jede reaktionsfähige Gruppe unbeeinflußt durch die andere reagiert. Setzt man die richtigen Werte der Differentialquotienten $d x / d t$ und $d y / d t$ aus dem Ansatz für zweistufige Reaktionen ein, so erbält man

$$
k=\frac{k_{1}(A-x)+k_{2}(x-y)}{2 A-x-y} .
$$

Differenziert man diese Gleichung nach $t$ und setzt für $d x / d t$ und $d y / d t$ wieder die Werte aus dem Ansatz für zweistufige Reaktionen ein, so erhält man

$$
\frac{d k}{d t}=\frac{\left(2 k_{2}-k_{1}\right)(A-x)(B-x-y)}{(2 A-x-y)^{2}}\left[k_{1}(A-y)-k_{2}(x-y)\right] .
$$

Aus den simultanen Differentialgleichungen der Stufenreaktion folgt ferner

$$
y=-\frac{1}{1-x} \cdot \frac{(A-x)^{\mathrm{x}}}{A^{\mathrm{x}-1}}+x+\frac{A-x}{1-\mathrm{x}},
$$


wo $x=k_{2} / k_{1}$. Führt man diesen Wert ein, so wird

$$
\frac{d k}{d t}=\frac{k_{1}\left(2 k_{2}-k_{1}\right)(B-x-y)(A-x)^{1+x}}{(2 A-x-y)^{2} A^{x-1}} .
$$

Das Zeichen dieses Ausdruckes wird von $2 k_{2}-k_{1}$ bestimmt. Ist $2 k_{2}>k_{1}$, so steigt $k$ mit fortschreitendem Umsatz, ist $2 k_{2}<k_{1}$, so sinkt $k$. Für $k_{1}=2 k_{2}$ bleibt $k$ während der ganzen Reaktion konstant; das muß ja herauskommen, weil dies die Bedingung für den scheinbaren einstufigen bimolekularen Reaktionsablauf ist. Bei großem Überschuß des Reagens gilt dasselbe; es ist nur $B$ statt $B-x-y$ zu setzen. In der Tat haben alle Autoren, welche einstufige bimolekulare Konstanten nach Äquivalenten (oder bei großem Überschuß des Reagens die entsprechenden monomolekularen Konstanten) gerechnet haben, entweder konstantes $k$ oder ein Fallen desselben gefunden. Das Fallen haben z. B. Petersen ${ }^{1}$ bei der direkten Esterbildung, Kailan ${ }^{2}$ bei der Veresterung mit alkoholischem Chlorwasserstoff, Reicher ${ }^{3}$ und Quartaroli ${ }^{4}$ bei der Verseifung mit wässerigen, $\mathrm{Hjelt}^{5}$ mit alkoholischen Basen beobachtet. Daraus ist zu schließen, daß in diesen Fällen $k_{2}$ kleiner ist als $k_{1} / 2$. Beim Versuch von Quartaroli wird allerdings das Sinken der Konstanten zum Teil durch den Dissoziationsrückgang der Base verursacht.

Aus Gleichung 24) folgt für $x=y=0 \quad k=k_{1} / 2$. Die Anfangswerte der einstufig nach Äquivalenten gerechneten Konstante nähern sich daher dem halben Wert der Konstante der ersten Stufe. Bei weit vorgeschrittenem Umsatz ( $x$ annähernd gleich $A$ ) nähert sich dagegen $k$ dem Wert der Konstanten der zweiten Stufe.

Setzt man die Anfangskonzentration des zweistufig reagierenden Stoffes in Molen ein, so setzt man bei der einstufigen Rechnung $d(x+y) / d t=k(A-x-y)(B-x-y)$. Dieser Ansatz hat eigentlich nur dann einen Sinn, wenn man $y$

1 Z. physik. Ch., 16, 405 (1895).

2 Z. physik. Ch., 85, 706 (1913).

3 Rec. trav chim., 4, 350 (1885).

4 Gazz. chim., 33, I, 497 (1903).

5 Ber. D. ch. G., 29, 1864 (1896). 
vernachlässigen kann. Dann bedeutet er die Berechnung der Konstanten der ersten Stufe bei Vernachlässigung der zweiten. Bei weit vorgeschrittenem Umsatz liefert er, wenn $B>A$, negative $k$-Werte, da $x+y$ größer wird als $A$. Bei diesem Ansatz ist

$$
k=\frac{k_{1}(A-x)+k_{2}(x-y)}{A-x-y} .
$$

Für $x=y=0$ ist $k=k_{1}$, entsprechend der bekannten Berechnung des Näherungswertes der Konstanten der ersten Stufe. Ein Näherungswert der Konstante der zweiten Stufe ist bei dieser Rechnungsart nicht zu gewinnen.

Bildet man in ähnlicher Weise wie früher $d k / d t$, so erhält man

$$
\begin{aligned}
& \frac{d k}{d t}= \frac{k_{1}^{2}(A-x)(B-x-y)}{(1-x)(A-x-y)^{2}} . \\
&\left\{(1-2 x) A+\left(\frac{A-x}{A}\right)^{x-1} .\right. \\
&\left.\cdot\left[-(1-x)(1-2 x)(A-x)+x^{2} A\right]\right\}
\end{aligned}
$$

Man kann auch $d k / d x$ bilden, wobei der aus den richtigen Differentialgleichungen folgende Wert $d y / d x=x(x-y) /(A-x)$ einzuführen ist. So erhält man

$$
\begin{aligned}
\frac{d k}{d x}= & \frac{k_{1}}{(A-x)(A-x-y)^{2}} . \\
& \cdot\left\{(A-x-y)\left[x A+x(1-x) x+(1-x)^{2} y\right]+\right. \\
& \left.+[x x+(1-x) y]^{2}\right\} .
\end{aligned}
$$

$B$ kommt in dieser Formel nicht vor, entsprechend dem von mir $^{1}$ in dem allgemeineren Fall der Glyzeridverseifung nachgewiesenen Satz, daß das Verhältnis der umgewandelten Mengen bei gleich weit vorgeschrittenem Umsatz von der Menge des den Umsatz bewirkenden Reagens unabhängig

1 Mon. f. Ch., 29, 122 (1908). 
ist. Indem man $y$ als Funktion von $x$ (Gleichung 25) einführt, erhält man aus der letzten Gleichung

$$
\begin{aligned}
& \frac{d k}{d x}= \frac{k_{1}}{(1-x)(A-x-y)^{2}} . \\
& \cdot\left\{(1-2 x) A+\left(\frac{A-x}{A}\right)^{x-1} .\right. \\
&\left.\cdot\left[-(1-x)(1-2 x)(A-x)+x^{2} A\right]\right\} .
\end{aligned}
$$

Diese Formel unterscheidet sich von Gleichung 28) nur um den Faktor $d t / d x=1: k_{1}(A-x)(B-x-y)$.

Für den Sonderfall $x=1$ werden die Formeln 28 und 30 unbrauchbar. Man erhält

$$
\frac{d k}{d t}=\frac{k_{1}^{2}(A-x)(B-x-y)}{(A-x-y)^{2}}\left[A-x-A l \frac{A-x}{A}\right],
$$

worin

$$
y=x-(A-x) l-\frac{A}{A-x} .
$$

Alle diese Formeln zeigen, daß $z$ u Beginn $k$ mit fortschreitendem Umsatz steigt. Denn man erhält für $t=x=$ $=y=0 \quad d k / d t=k_{1}^{2} x B, d k / d x=k_{1} x / A$. Für $x=A$ wird $d k / d t=0 . d k / d x$ hat dagegen verschiedene Werte je nach den Werten von $x$. Bei $x=A$ ist für $x>1 d k / d x=$ $=k_{1}(1-2 x) /(1-x) A$, also endlich und positiv; dagegen ist für $x \equiv 1 d k / d x$ positiv unendlich.

$\mathrm{Da}$ für $k_{1}>2 k_{2}$ die nach Äquivalenten gerechnete Konstante einen fallenden, die nach Molen einen steigenden Gang hat, kann man in diesem Fall durch Verwendung beider Rechnungsarten einen unteren und einen oberen Grenzwert für die Geschwindigkeitskonstante der ersten Stufe erhalten, falls die Versuche genau genug sind.

Aus Gleichung 27) sieht man, daß $k$ für $A-x-y>0$ positiv ist, da $A-x$ und $x-y$ immer positiv sind, ferner, daß es für $A-x-y=0$, also, wenn die Titeränderung in $\ddot{\text { qqui- }}$ valenten gleich ist der Anfangskonzentration des Ausgangsstoffes in Molen, durch unendlich geht und für $A-x-y<0$ 
negativ wird, wobei mit fortschreitender Zeit der Wert von $-\infty$ bis Null geht. Letzterer Wert wird erreicht, wenn $x=y=A$. Vollständig können daher diese Werte nur durchlaufen werden, wenn $B \equiv 2 A$.

Einen genaueren Einblick in den Verlauf der $k$-Werte bekommt man am besten aus Zahlenbeispielen. Diese lassen sich für willkürlich gewählte $x$ unschwer rechnen, da $y$ als Funktion von $x$ bekannt ist (Gleichung 25,32) und die Formel für $k$ keine anderen veränderlichen Größen enthält. Ich setze einige Beispiele her.

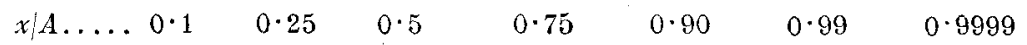

$$
\begin{aligned}
& \%=0 \cdot 01
\end{aligned}
$$

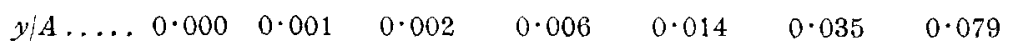



$$
\begin{aligned}
& \mathrm{x}=\mathbf{0} \cdot \mathbf{1} \\
& \begin{array}{rrrrrrrr}
y / A \ldots \ldots & \ldots .000 & 0.004 & 0.019 & 0.060 & 0.128 & 0.300 & 0.558
\end{array} \\
& \begin{array}{llllllll}
k / k_{1} \ldots \ldots & 1.012 & 1.038 & 1.139 & 1.682 & -6.238 & -0.273 & -0.079
\end{array} \\
& x=\mathbf{0} \cdot 5 \\
& \begin{array}{lllllllll}
y: A \ldots .0 .003 & 0.018 & 0.085 & 0.250 & 0.468 & 0.810 & 0.980
\end{array} \\
& \begin{array}{lllllllll}
k_{i} / k_{1} & \ldots & 1.057 & 1.183 & 1.705 & \infty & -0.860 & -0.125 & -0.010
\end{array} \\
& x=1 \cdot 0
\end{aligned}
$$

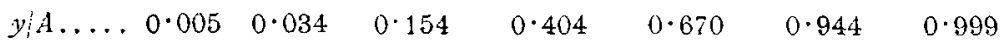

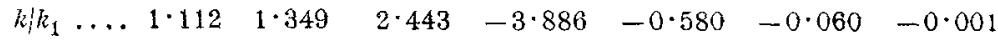

$$
\begin{aligned}
& \mathrm{x}=\mathbf{1 1 \cdot 0}
\end{aligned}
$$

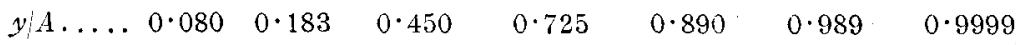

$$
\begin{aligned}
& \begin{array}{lllllllll}
k / k_{1} & \ldots & 1.370 & 2.623 & 21.01 & -1.105 & -0.266 & -0.022 & -0.0002
\end{array}
\end{aligned}
$$

Die Zahlen zeigen den durch die Formeln geforderten Gang. In dem gewöhnlichen Fall, wo $x$ klein ist, erhält man aus den Anfangsbeobachtungen gute Näherungswerte für $k_{1}$, und zwar, soweit meine Erfahrung reicht, bessere als bei der Rechnung nach Äquivalenten. Je kleiner $x$, desto länger bleibt $k$ ungefähr konstant. Doch beginnt das stärkere Ansteigen schon, wenn das Produkt der zweiten Stufe noch in sehr geringem $\mathrm{Maß}$ gebildet ist (z. B. $x=0.01, y / A=0.014, k / k_{1}=1.26$ ). Bei großem $x$ können gute Näherungswerte von $k_{1}$ nicht er: halten werden. 
Mit den Forderungen der Theorie bezüglich der "nach Molen gerechneten einstufigen Konstante stehen die Beobachtungen nicht immer im Einklang. $Z_{w}$ ar ist das Ansteigen dieser Konstanten von Neelmeier ${ }^{1}$ bei der Verseifung mit alkoholischem Alkali im Verhältnis $1 \mathrm{Mol}: 2 \mathrm{Mol}$ immer und beim Verhältnis 1:1 Mol an zwei Estern beobachtet worden; auch Horn ${ }^{2}$ hat dies am Bernsteinsäureester in 50 prozentigem Alkohol beobachtet. Es steht auch nicht im Widerspruch mit der Theorie, daß beim Verhältnis $1: 1 \mathrm{Mol}$ Conrad und Brückner ${ }^{3}$ sowie Neelmeier in mehreren Fällen (letzterer auch ausnahmslos bei der Verseifung mit 0.5 Molen Alkali) gute Konstanz des nach Molen berechneten einstufigen bimolekularen Geschwindigkeitskoeffizienten beobachtet haben. In diesen Fällen kann $x / A$ höchstens den Wert $0 \cdot 5$, beziehungsweise 0.25 erreichen; in diesem Bereich muß bei kleinem $x k$ nahezu konstant sein. Aber es steht im Widerspruch mit der Theorie, daß Neelmeier beim Molenverhältnis $1: 1$ bisweilen, ferner Goldschmidt und Scholz ${ }^{4}$ sowie J. Meyer ${ }^{5}$ bei der Verseifung mit wässerigem Alkali in den Molenverhältnissen $1: 1$ und $1: 2$ durchwegs fallende Konstanten erhalten haben. Es müssen also entweder die theoretischen Voraussetzungen nicht erfüllt gewesen sein oder systematische Versuchsfehler vorliegen. Die theoretischen Voraussetzungen wären $z$. B. nicht erfüllt, wenn die Geschwindigkeit nicht proportional der Gesamtkonzentration des Alkalis wäre. Das ist für alkoholische Lösungen wahrscheinlich zutreffend, aber nicht für die von J. Meyer untersuchten verdünnten wässerigen Lösungen. In der Tat erklären Goldschmidt und Scholz ihren Befund durch die systematischen Fehler der angewendeten Titrationsmethode.

\footnotetext{
I Diss. Halle, 1902.

2 Diss. Leipzig, 1908.

3 Z. physik. Ch., 7, 287 (1891).

4 Ber. D. ch. G., 36, 1333 (1903).

5 Z. physik. Ch., 67, 278 (1909).
} 


\section{Zusammenfassung.}

1. Es wird eine Übersicht der Entwicklung der Lehre von den Stufenreaktionen insbesondere bezüglich der Bildung und Verseifung der Dicarbonsäureester und eine Zusammenstellung der Formeln für zweistufige Reaktionen bei großem Überschuß des Reagens mit und ohne Auftreten isomerer Zwischenprodukte für den allgemeinen Fall und den des Auftretens des scheinbar monomolekularen Reaktionsablaufes gegeben.

2. Daß die Bildung und Verseifung der Dicarbonsäureester stufenweise erfolgt, folgt schon aus der Darstellbarkeit der Estersäuren bei diesen Reaktionen. Die chemische Kinetik hat in diesem Fall nur die Aufgabe, zu prüfen, ob der Reaktionsverlauf durch Stufenreaktionen restlos darstellbar ist.

3. Proportionalität $z$ wischen Zahl der reaktionsfähigen Gruppen in der Molekel und Geschwindigkeitskonstanten der Stufenreaktionen tritt auch dann häufig nicht ein, wenn die Abweichungen von der Proportionalität nicht durch elektrostatische Wirkungen der Ionen erklärbar sind.

4. Es wird der Gang untersucht, den die bimolekular einstufig gerechneten Geschwindigkeitskonstanten zeigen müssen, wenn eine bimolekulare zweistufige Reaktion vorliegt. Der Gang ist verschieden, je nachdem die Anfangskonzentration des stufenweise umgewandelten Stoffes in Äquivalenten oder in Molen eingesetzt wird. Hierbei ergeben sich Regeln für die Gewinnung von Näherungswerten der Konstanten der Stufenreaktionen durch einstufige Rechnung. 